\title{
Quota setting and enforcement choice in a shared fishery
}

\author{
Aaron Hatcher* \\ Portsmouth Business School, \\ University of Portsmouth, United Kingdom \\ Linda Nøstbakken ${ }^{\dagger}$ \\ Norwegian School of Economics, Bergen, Norway \\ *University of Portsmouth, \\ Richmond Building, Portland Street, \\ Portsmouth PO1 3DE, United Kingdom \\ Tel. $+44(0) 2392848510$ \\ E-mail: aaron.hatcher@port.ac.uk \\ ${ }^{\dagger}$ Norwegian School of Economics (NHH), \\ Helleveien 30, \\ 5045 Bergen, Norway \\ Tel: +4755959134 \\ E-mail: linda.nostbakken@nhh.no
}

This work was supported by the Research Council of Norway under its Oceans and the Coastal Areas program (Grant no. 185151). The authors would like to thank two anonymous referees for their helpful comments and suggestions. 


\section{Introduction}

The problem of the cooperative (or indeed non-cooperative) management of shared renewable resources, such as transboundary fish stocks, has received a considerable amount of attention in the literature, primarily from a game theoretic perspective (see, for example, Kaitala and Lindroos 2007, Lindroos 2008, Munro 2009, Hannesson 2011, Long 2011, 2012, Lindroos and Munro 2013). In general, analysis has focused on the determination of optimal harvest shares and the possibilities for cooperative agreement on harvests by participating countries. In common with most theoretical models of fishery exploitation, either harvest itself, or harvest as a function of fishing "effort", is taken as the control variable. In practice, however, national administrations are only able to control harvest indirectly, by introducing management measures (such as quotas) and then expending enforcement effort in order to achieve a degree of compliance by their fishing industries. Given that enforcement is costly, and there is, therefore, a social cost to controlling harvest, the need for enforcement inevitably implies second-best solutions in which optimal harvests differ from those which would hold were enforcement perfect and costless. ${ }^{1}$

It is also apparent that, in the case of many shared fish stocks, for better or worse international agreements do exist on harvest shares, at least in principle. Once such agreements are reached, moreover, they tend to endure, since they are politically costly to renegotiate. A good example is provided by the Atlantic and North Sea fisheries of the European Union (EU). ${ }^{2}$ Here, fixed shares of TACs ("total allowable catches") were

1 This mirrors, but is not identical with, the more general observation that perfect regulatory enforcement is unlikely to be optimal if enforcement is costly (Sutinen and Andersen 1985). The distinction stems from the fact that here we are not trying to achieve compliance per se but rather an efficient level of harvest.

${ }^{2}$ A notable exception is the Northeast Atlantic mackerel fishery, which is shared between the EU, Norway, the 
agreed in 1983 after years of difficult negotiations (see Holden and Garrod 1994). Since then, the shares have remained more or less constant (according to the so-called principle of "relative stability") as no EU Member State has wanted to reopen negotiations. The fundamental problems for resource management in such cases are, firstly, the setting of an annual TAC or TACs to be divided amongst participating countries as national quotas, and secondly, compliance with those quotas by national fishing fleets, which depends upon countries' individual enforcement efforts. These real-world problems form the motivation for the investigations in this paper.

The paper builds upon earlier work by Beard and Nøstbakken (2010) which analysed a dynamic noncooperative game in enforcement between two countries. Here, we consider both enforcement and the setting of the TAC in a model of a single shared (transboundary) fish stock exploited by two countries which have previously reached agreement on their percentage shares of the TAC. ${ }^{3}$ Once the TAC is determined, therefore, each country's quota is fixed and the problem for the country is then to choose a level of costly enforcement effort to apply to its fishing fleet in order to maximise national economic benefits while securing what it considers to be an acceptable degree of compliance with the quota (which we always assume to be constraining on harvest). Given the national quota and the level of enforcement, the fleets then respond rationally with a privately optimal harvest level. We assume that the country knows this harvest response function when it chooses a level of enforcement effort and hence national harvest and enforcement are jointly determined. We therefore treat national enforcement choice as essentially myopic,

Faroe Islands and Iceland. In this case, all parties agree on the ICES-recommended TAC, but due in part to changing migration patterns there was profound disagreement over quota shares.

3 We leave to one side the question of how such an agreement was reached. 
with countries' concerns for the long term conservation of the stock focused primarily on the determination of the TAC. Nevertheless, we compare the countries' enforcement choices with the socially efficient levels of enforcement, given the quotas in force (this is equivalent to the cooperative outcome for a dynamic game in quota enforcement).

We then go on to consider the setting of an efficient TAC for the fishery, given the agreed national TAC shares and the enforcement and harvest choices of the participating countries' administrations and their fishing fleets. At first sight, the problem seems straightforward enough. We find, however, that there are two sources of inefficiency in the optimal management of a shared resource in this way. The first stems from the likely sub-optimal allocation of the TAC between countries. In principle at least, this could simply be solved by adjustments in the quota shares. The second source of inefficiency lies in the (assumed) desire of countries to achieve a degree of compliance by their fishing fleets. Although, for a given quota, it is possible for national enforcement levels to be efficient, this does not hold when we are trying to achieve optimal management of the fishery using the TAC. Because enforcement is costly, a fully efficient ("first-best") solution to management by TAC would be to set a TAC at a very low level and then to relax enforcement to allow a relatively large margin of non-compliance (and hence an efficient level of harvest). This is not possible, however, if countries want to limit non-compliance.

We illustrate this problem within a set of simple numerical simulations for the (most tractable) case of symmetrical countries and equal TAC shares. We compare the efficient levels of harvest, enforcement (given a TAC) and TAC itself (given a range of countries' implicit preferences for compliance). 
Lastly, we consider the incentives for countries to seek increases in the TAC during the annual decision-making process in which a recommended TAC must be agreed upon by participant countries. Interestingly, we find that countries which care more about quota compliance have a greater incentive to seek a higher TAC, since they gain more from reduced enforcement costs as the TAC is increased.

The paper is structured as follows. We begin by developing the non-compliant industry harvest model and then examine the efficient enforcement choices for individual countries, given their national quotas (TAC shares). The countries' myopic enforcement choices are compared in the following section. In Section 4 we turn to the problem of setting an efficient TAC for an international fishery with imperfect national enforcement and then consider the incentives for participating countries to bid up an arbitrary TAC during annual negotiations. Section 5 presents the results of a numerical simulation, while a final section concludes.

\section{Model and preliminaries}

We model a single species fishery exploited by fishing vessels from $N$ countries which are party to an international agreement on percentage shares of an annual TAC. Without loss of generality, in what follows we consider the situation where $N=2$.

\subsection{The industry's harvest response function}

We begin by modelling the harvest response (reaction function) of the industry in Country 
$i$ to the agreed national quota (TAC share) and the national level of enforcement effort. Here, following Beard and Nøstbakken (2010), enforcement effort is normalised to the probability that a particular firm is inspected and fined for landing over-quota fish (which implies an underlying linear relationship between enforcement effort and the probability of inspection and sanction). For further simplicity, and to abstract from the problem of allocative efficiency at the national level, we represent the industry in each country by a single (price-taking) firm. The firm chooses a level of harvest to maximise (expected) per period profits, net of fines for non-compliance, as

$$
\max _{h} E\left(\pi_{i}\left(h_{i} ; x\right)-\phi_{i} f_{i}\left(h_{i} ; q_{i}\right)\right) \equiv p h_{i}-c_{i}\left(h_{i} ; x\right)-\phi_{i} f_{i}\left(h_{i} ; q_{i}\right),
$$

where $h_{i}$ is the harvest, $p$ is the (exogenous) market price, $c_{i}(\cdot)$ are harvesting costs, $x$ is the stock size (which the firm takes as given in the short run), $\phi_{i} \in[0,1]$ is the country's chosen level of enforcement effort (probability of detection), $f_{i}$ is a monetary fine and $q_{i}$ is the national quota. To give a specific form to the firm's objective function, let

$$
E\left(\pi_{i}(\cdot)-\phi_{i} f_{i}(\cdot)\right) \equiv p h_{i}-\frac{c_{i}}{2 x} h_{i}^{2}-\phi_{i} f_{i}\left[\frac{h_{i}-q_{i}}{q_{i}}\right], \quad h_{i} \geq q_{i},
$$

where $c_{i}$ is a harvesting cost parameter ${ }^{4}$ and $f_{i}$ is a fine rate. Note that here the expected penalty for landing over-quota fish is a linear function of the relative quota violation, which ensures that the firm's optimal per period harvest is dependent upon the size of the quota $q_{i} \cdot{ }^{5}$ The necessary first order condition for an (expected) profit-maximising level

4 Without loss of generality, we choose the simplest concave in harvest profit function, which assumes quadratic costs.

5 Otherwise, the model could equally well represent a vessel operating under a management system based upon taxes, rather than quotas. Note that we could, alternatively, have an expected penalty which is a non-linear function of the level violation $[h-q]$, but the functional form used here is less cumbersome. Hatcher (2005, 2012) considers the intuition for relative violation arguments in expected penalty functions and examines the implications for modelling quota compliance in natural resource industries. 
of harvest is

$$
p-\frac{c_{i}}{x} h_{i}^{*}-\phi_{i} \frac{f_{i}}{q_{i}}=0,
$$

where we assume that $h_{i}^{*}>q_{i}$ and hence $\phi_{i} f_{i} / q_{i}>0$, that is, the quota is less than the industry's capacity output and there is non-compliance as a result. Capacity output $\bar{h}_{i}$ is therefore defined as the output the industry would choose in the absence of regulation, where $\phi_{i} f_{i} / q_{i}=0$ and hence

$$
p-\frac{c_{i}}{x} \bar{h}_{i}=0
$$

Given a quota $q_{i}$, however, from (3) we can find the industry's optimal harvest response to the country's choice of enforcement effort $\phi_{i}$ as

$$
h_{i}^{*}\left(\phi_{i} ; q_{i}, x\right)=\frac{x}{c_{i}}\left[p-\phi_{i} \frac{f_{i}}{q_{i}}\right] .
$$

From (5) we can see that, as would be expected, the industry's optimal harvest response $h_{i}^{*}($.$) is increasing in the stock size, the market price and the size of the quota and$ decreasing in harvest costs, enforcement effort and the fine rate. Note, in particular, that

$$
h_{i \phi}^{*} \equiv \frac{\partial h_{i}^{*}(\cdot)}{\partial \phi_{i}}=-\frac{x f_{i}}{c_{i} q_{i}}<0
$$

\subsection{Efficient enforcement choice}

Before proceeding to examine the individual countries' myopic enforcement choices, we consider, as a benchmark, the optimal level of enforcement for each country if a social planner were responsible for the decision, given an agreed annual quota pair $q_{i}, i=1,2$. This is equivalent to the cooperative outcome for two countries interested in maximis- 
ing their joint social surplus under the same quota constraints. The specific objective in this case is to employ enforcement effort in both countries so as to maximise the total discounted flow of industry harvesting profits (including any fines, since these are simply transfers) less the costs of enforcement, subject to the (known) industry harvest responses and the dynamics of the stock, given the national quotas in force. ${ }^{6}$ Omitting time arguments for clarity, the objective functional is

$$
\begin{gathered}
\max _{\phi_{1}, \phi_{2}} \int_{0}^{\infty} e^{-r t}\left\{\sum_{i=1}^{2}\left[\pi_{i}\left(h_{i}^{*}(\cdot)\right)-\omega_{i}\left(\phi_{i}\right)\right]\right\} d t \\
\text { s.t. } \quad \dot{x}=g(x)-\sum_{i=1}^{2} h_{i}^{*}(\cdot),
\end{gathered}
$$

where $\omega_{i}\left(\phi_{i}\right)$ is the cost of enforcement in Country $i, g(x)$ is the natural growth function for the stock and $r$ is an appropriate discount rate. Letting $\omega_{i}^{\prime}\left(\phi_{i}\right) \equiv \omega_{i}$, a constant marginal cost of enforcement, the corresponding (current value) Hamiltonian is

$$
\mathcal{H}^{c}(\cdot) \equiv \sum_{i=1}^{2}\left[\pi_{i}(\cdot)-\omega_{i} \phi_{i}\right]+\lambda\left[g(x)-\sum_{i=1}^{2} h_{i}^{*}\right],
$$

where $\lambda$ is the Lagrange multiplier on the stock constraint. ${ }^{7}$ The necessary conditions for maximising the Hamiltonian with respect to $\phi_{i}>0$ are

$$
\mathcal{H}_{\phi}^{c} \equiv\left[\pi_{i h}-\lambda^{*}\right] h_{i \phi}^{*}-\omega_{i}=0, \quad i=1,2
$$

where

$$
\pi_{i h} \equiv \frac{\partial \pi_{i}(\cdot)}{\partial h_{i}}=p-\frac{c_{i}}{x} h_{i}^{*}, \quad i=1,2 .
$$

Substituting for $h_{i}^{*}$ and $h_{i \phi}^{*}$ using (5) and (6), we can see that the efficient level of enforce-

6 We assume an appropriately long period of time: for the sake of argument we take an infinite time horizon.

7 By specifying constant harvesting cost parameters $c_{i}$, we make the implicit assumption that industry capacity in each country remains fixed. Arguably, a social planner aiming to maximise the economic value of the fishery would choose a combined level of capacity (capital) that is optimal for the TAC, but we ignore this possibility here. 
ment effort for Country $i$ is where

$$
\phi_{i}^{*}=\frac{q_{i}}{f_{i}}\left[\lambda^{*}-\omega_{i} \frac{c_{i} q_{i}}{x f_{i}}\right]>0
$$

Note that $\phi_{i}^{*}>0$ assumes that quotas are set at levels at which a positive level of costly enforcement is optimal, which implies that the (second-best) harvest is greater than the quota for each country, though less than industry capacity.

Rearranging (9), the first order condition for $\phi_{i}^{*}$ implies

$$
\lambda^{*}=\pi_{i h}-\frac{\omega_{i}}{h_{i \phi}^{*}}>0, \quad i=1,2,
$$

where, recall, $h_{i \phi}^{*}<0$. Thus, the shadow price for the stock is greater than the marginal profit from harvesting in Country $i$. The difference is due to the (marginal) cost of enforcement, or, more accurately here (since the control variables reduce harvest), the marginal reduction in the cost of controlling the harvest through enforcement. Notice from (6) that this is increasing in the size of the quota $q_{i}$. This is because, all else equal, in order to achieve any given harvest level, a larger quota implies a smaller violation which is more costly to attain in terms of enforcement effort. We can also see from (12) that if the marginal cost of enforcement effort $\omega_{i}$ is higher in one country than in the other, then, all else equal, marginal profit (in harvest) must be smaller in that country and hence the level of harvest greater. Thus, for a given quota pair, enforcement effort, when allocated optimally, is concentrated in the country where it is least costly to deploy.

The corresponding stock condition is

$$
\dot{\lambda}-r \lambda^{*}=-\mathcal{H}_{x}^{c} \equiv-\sum_{i=1}^{2} \pi_{i x}-\lambda^{*}\left[g_{x}-\sum_{i=1}^{2} h_{i x}^{*}\right],
$$


where

$$
\pi_{i x} \equiv \frac{\partial \pi_{i}(\cdot)}{\partial x}=\left[p-\frac{c_{i}}{x} h_{i}^{*}\right] h_{i x}^{*}+\frac{c_{i}}{2 x^{2}} h_{i}^{* 2}>0
$$

and

$$
h_{i x}^{*} \equiv \frac{\partial h_{i}^{*}(\cdot)}{\partial x}=\frac{1}{c_{i}}\left[p-\phi_{i}^{*} \frac{f_{i}}{q_{i}}\right]>0 .
$$

If we assume that a steady state is attained $(\dot{x}=\dot{\lambda}=0)$, then we can find

$$
\lambda^{*}=\frac{\sum_{i=1}^{2} \pi_{i x}}{r-\left[g_{x}-\sum_{i=1}^{2} h_{i x}^{*}\right]} .
$$

Substituting for $\lambda^{*}$ using (12) and rearranging terms, we obtain

$$
r=\left[g_{x}-\sum_{i=1}^{2} h_{i x}^{*}\right]+\frac{\sum_{i=1}^{2} \pi_{i x}}{\pi_{i h}-\omega_{i} / h_{i \phi}^{*}}, \quad i=1,2 .
$$

Given the TAC and hence the national quotas $q_{i}$, each country's steady state harvest under optimal enforcement satisfies the "golden rule" shown in (17). The first (bracketed) term on the RHS of the equation is the (adjusted) marginal growth rate of the stock while the second term on the RHS is the "marginal stock effect", i.e., the marginal value of the stock in situ (which would be zero if harvesting costs were independent of stock size). Equation (17) differs from more familiar versions of the golden rule ${ }^{8}$ in the inclusion of the $h_{i x}^{*}$ and $\omega_{i} / h_{i \phi}^{*}$ terms, which appear because the control variables are the quantities of enforcement effort, rather than harvest levels directly.

\subsection{Noncooperative outcome}

The noncooperative dynamic outcome for Country $i$ as an open-loop Nash equilibrium (OLNE) can be found fairly straightforwardly. ${ }^{9}$ In this case, we have the first order

8 See Clark and Munro (1975): sometimes referred to as the "fundamental equation of renewable resources" (e.g., Conrad 1999).

9 The alternative "feedback" (closed-loop) Markov-perfect Nash equilibrium (MPNE), in which enforcement choices 
condition for enforcement effort $\phi_{i}>0$ as

$$
\left[\pi_{i h}-\lambda_{i}^{*}\right] h_{i \phi}^{*}-\omega_{i}=0 \quad \Rightarrow \quad \lambda_{i}^{*}=\pi_{i h}-\frac{\omega_{i}}{h_{i \phi}^{*}}>0
$$

while the stock condition (taking the harvest by Country $j$ as exogenous) is simply

$$
\dot{\lambda}_{i}-r_{i} \lambda_{i}^{*}=-\pi_{i x}-\lambda_{i}^{*}\left[g_{x}-h_{i x}^{*}\right]
$$

Solving for $\lambda_{i}^{*}$, we obtain the steady state solution (assuming there is one) as $\phi_{i}^{*}$ satisfying

$$
r_{i}=\left[g_{x}-h_{i x}^{*}\right]+\frac{\pi_{i x}}{\pi_{i h}-\omega_{i} / h_{i \phi}^{*}},
$$

similarly for Country $j$.

Equation (20) is formally identical to (17), except that in the noncooperative case each country does not take into account the stock effect of its own harvest on the harvesting costs of the other country.

\section{Country-specific (myopic) enforcement}

In practice, we assume, when each country is unilaterally deciding upon a level of enforcement effort, concern about the long term value of the stock is effectively relegated to the international TAC setting process (see Section 4): once an annual quota pair $q_{i}, i=1,2$, has been agreed, countries behave myopically and maximise only current period social benefits. Nevertheless, we assume that each country has some concern for adhering to its agreed quota, which we represent by a perceived social cost attached to over-quota harvesting by the country's fleet. ${ }^{10}$

depend upon the stock variable $x$, cannot be found analytically for our model. In general feedback solutions are very difficult to find for dynamic fishery games, except under rather special restrictions (see Long 2011).

10 This characterisation of national perceptions and priorities is not unrealistic within the EU, for example, where the 
Given this, Country i's objective function is

$$
\max _{\phi} \pi_{i}\left(h_{i}^{*}(\cdot)\right)-\omega_{i}\left(\phi_{i}\right)-\delta_{i}\left(h_{i}^{*}(\cdot) ; q_{i}\right),
$$

where $\delta_{i}($.$) is a social "damage" (or disutility) function for exceeding the quota (measured$ in monetary units for convenience). In specific form, we can write the country's problem as

$$
\max _{\phi}\left[p h_{i}^{*}(\cdot)-\frac{c_{i}}{2 x} h_{i}^{*}(\cdot)^{2}\right]-\omega_{i} \phi_{i}-\delta_{i}\left[\frac{h_{i}^{*}(\cdot)-q_{i}}{q_{i}}\right],
$$

where the last term, notice, implies that the country cares about the degree to which the quota is exceeded, rather than the nominal violation. The first order condition for the optimal level of enforcement effort $\phi_{i}^{*}$ is then

$$
\left[p-\frac{c_{i}}{x} h_{i}^{*}-\frac{\delta_{i}}{q_{i}}\right] h_{i \phi}^{*}-\omega_{i}=0
$$

Substituting for $h_{i}^{*}$ and $h_{i \phi}^{*}$ using (5) and (6) and rearranging, we obtain

$$
\phi_{i}^{*}=\frac{q_{i}}{f_{i}}\left[\frac{\delta_{i}}{q_{i}}-\omega_{i} \frac{c_{i} q_{i}}{x f_{i}}\right]
$$

which we can see is exactly analogous to (11). Here, though, since $\delta_{i}$ is a parameter describing the strength of the country's desire to comply with its quota, the interpretation is that a positive level of enforcement effort requires that $\delta_{i} / q_{i}>\omega_{i} c_{i} q_{i} / x f_{i}$. Since $\omega_{i} c_{i} q_{i} / x f_{i}=-\omega_{i} / h_{i \phi}^{*}$, the marginal social cost to the country of over-quota landings must exceed the (saving in) enforcement costs of a marginal increase in the over-quota catch: otherwise, the country will not undertake any costly enforcement.

For a given pair of quotas $q_{i}$, an efficient level of enforcement would, as we might expect, result from $\delta_{i} / q_{i}=\lambda^{*}$ in each country. In effect, for a given quota the parameter $\delta_{i}$ at Community, rather than Member State, level. 
represents the country's own shadow pricing of the stock. If a country cares too little about over-quota catches, the total level of enforcement will be sub-optimal and the total level of harvest will be excessive. On the other hand, we also have the perverse possibility that, if a country cares too much about over-quota landings, it will employ too much costly enforcement effort compared to the efficient level. It should be apparent, however, that what constitutes too much or too little enforcement, relative to the social optimum, depends upon the magnitude of the quotas set. If, for example, the quotas are relatively low, then the optimal amount of enforcement will be less than if the quotas were set at a higher level (see Section 4). Under optimal enforcement, of course, the values of $\phi_{i}^{*}$ and $\lambda^{*}$ are jointly determined.

We can see from (24) that, all else equal, Country $i$ 's choice of enforcement effort $\phi_{i}^{*}$ is increasing in its disutility "rate" for over-quota catches $\delta_{i}$ and decreasing in the size of the quota $q_{i}$ and the cost of enforcement $\omega_{i}$. We may recall that a standard result from environmental enforcement models is that less enforcement effort is required in order to achieve a given target (e.g., level of harvest) if the fine is increased, since expected penalties are a product of the fine and the subjective probability of detection and sanction (see, for example, Sutinen and Andersen, 1985). Here, though, because there are social benefits as well as costs to violation (through increased industry profits and reduced enforcement costs), the net impact of altering the fine rate $f_{i}$ on the optimal level of enforcement is ambiguous. Increasing the fine, all else equal, increases the effectiveness of enforcement in reducing (over-quota) harvest, but while this reduces the disutility from over-quota 
catches, it also reduces industry profits. From

$$
\frac{\partial \phi_{i}^{*}}{\partial f_{i}}=\frac{q_{i}}{f_{i}^{2}}\left[2 \omega_{i} \frac{c_{i} q_{i}}{x f_{i}}-\frac{\delta_{i}}{q_{i}}\right] \gtreqless 0,
$$

we can see that if $\delta_{i} / q_{i}>2 \omega_{i} c_{i} q_{i} / f_{i} x$ then increasing the fine reduces the optimal level of enforcement effort. If increasing the fine (which effectively reduces the cost of controlling harvest) reduces the social costs of (over-quota) harvesting more than the benefits, we would expect enforcement effort to be relaxed. Conversely, if increasing the fine reduces the benefits of harvesting more than the social costs, we would expect enforcement to be increased.

\section{Setting the total allowable catch (TAC)}

Having established the (myopic) enforcement effort response of each country to a national quota, given the harvest response of its fishing industry, we now turn to the problem of setting a TAC when the shares of the TAC used in calculating the national quotas are fixed and individual countries are responsible for enforcing quotas upon their fishing industries. We begin by examining the optimal TAC in a dynamic setting and then consider the incentives for countries to "bid up" an arbitrary TAC during a political process.

\subsection{Optimal TAC setting with fixed quota shares}

We let an omniscient central planner set an annual TAC $(Q)$ for the fishery which translates into national quotas $q_{i} \equiv \gamma_{i} Q$ for the two countries, where $\gamma_{1}+\gamma_{2}=1$ are the TAC shares that have previously been agreed. Using (24) and (5), we can rewrite the 
individual countries' enforcement choices in terms of $Q$ as

$$
\phi_{i}^{*}(Q ; x)=\frac{\gamma_{i} Q}{f_{i}}\left[\frac{\delta_{i}}{\gamma_{i} Q}-\gamma_{i} Q \frac{\omega_{i} c_{i}}{x f_{i}}\right]>0, \quad i=1,2,
$$

given their industries' harvest response functions

$$
h_{i}^{*}\left(\phi_{i}^{*} ; x\right)=\frac{x}{c_{i}}\left[p-\phi_{i}^{*}(Q) \frac{f_{i}}{\gamma_{i} Q}\right]>0, \quad i=1,2 .
$$

Substituting (26) into (27) and rearranging, we obtain an expression for industry harvest as a function of $Q$ (given a stock size $x$ ) as

$$
h_{i}^{*}\left(\phi_{i}^{*}(Q) ; x\right)=\frac{x}{c_{i}}\left[p-\frac{\delta_{i}}{\gamma_{i} Q}\right]+\gamma_{i} Q \frac{\omega_{i}}{f_{i}}, \quad i=1,2,
$$

where, recall, we assume that $\phi_{i}^{*}()>$.0 and hence $\bar{h}_{i}>h_{i}^{*}(\cdot)>\gamma_{i} Q$, i.e., the industry in each country is non-compliant but harvests at less than capacity due to the enforcement efforts of a national regulator. Note that here

$$
h_{i \delta}^{*} \equiv \frac{\partial h_{i}^{*}}{\partial \delta_{i}}=-\frac{x}{c_{i} \gamma_{i} Q}<0,
$$

while

$$
h_{i Q}^{*} \equiv \frac{\partial h_{i}^{*}}{\partial Q}=\frac{x \delta_{i}}{c_{i} \gamma_{i} Q^{2}}+\gamma_{i} \frac{\omega_{i}}{f_{i}}>0,
$$

so that industry harvest is a concave function of the TAC as we approach capacity output $\bar{h}_{i}$

Omitting time arguments as before, the planner's objective functional is now

$$
\begin{gathered}
\max _{Q} \int_{0}^{\infty} e^{-r t}\left\{\sum_{i=1}^{2}\left[\pi_{i}\left(h_{i}^{*}(\cdot)\right)-\omega_{i} \phi_{i}^{*}(\cdot)\right]\right\} d t \\
\text { s.t. } \quad \dot{x}=g(x)-\sum_{i=1}^{2} h_{i}^{*}(\cdot)
\end{gathered}
$$

and the corresponding (current value) Hamiltonian is

$$
\mathcal{H}^{c}(\cdot) \equiv \sum_{i=1}^{2}\left[\pi_{i}\left(h_{i}^{*}(\cdot)\right)-\omega_{i} \phi_{i}^{*}(\cdot)\right]+\lambda\left[g(x)-\sum_{i=1}^{2} h_{i}^{*}(\cdot)\right] .
$$


The first order condition for a maximising choice of $Q>0$ is

$$
\mathcal{H}_{Q}^{c} \equiv \sum_{i=1}^{2}\left[\left[\pi_{i h}-\lambda^{*}\right] h_{i Q}^{*}-\omega_{i} \phi_{i Q}^{*}\right]=0,
$$

where, as before,

$$
\pi_{i h} \equiv \frac{\partial \pi_{i}(\cdot)}{\partial h}=p-\frac{c_{i}}{x} h_{i}^{*}(\cdot), \quad i=1,2 .
$$

Substituting in (33) for $h_{i}^{*}(\cdot)$ and $h_{i Q}^{*}$ using (28) and (30), together with

$$
\phi_{i Q}^{*} \equiv \frac{\partial \phi_{i}^{*}(\cdot)}{\partial Q}=-2 \gamma_{i}^{2} Q \frac{\omega_{i} c_{i}}{x f_{i}^{2}}<0,
$$

after some rearrangement we obtain the optimality condition in the form

$$
\sum_{i=1}^{2}\left[\frac{x \delta_{i}}{c_{i} \gamma_{i} Q^{* 2}}\left[\frac{\delta_{i}}{\gamma_{i} Q^{*}}-\lambda^{*}\right]+\gamma_{i} \frac{\omega_{i}}{f_{i}}\left[\gamma_{i} Q^{*} \frac{\omega_{i} c_{i}}{x f_{i}}-\lambda^{*}\right]\right]=0
$$

In order to interpret equation (36), consider the case where the two countries are identical in every respect, including in their quota shares $\gamma_{i}$ : this ensures that the TAC is efficiently allocated. If we have positive levels of national enforcement effort $\phi_{i}^{*}$ (as we assume), we can see from (26) that we must have

$$
\frac{\delta_{i}}{\gamma_{i} Q^{*}}>\lambda^{*}>\gamma_{i} Q^{*} \frac{\omega_{i} c_{i}}{x f_{i}}
$$

Recall that, for any given quota $q_{i}$, an efficient level of myopic national enforcement would require that $\delta_{i} / q_{i}=\lambda^{*}$. Here though, because the non-compliance "disutility" parameter $\delta_{i}$ does not represent a real resource cost, enforcement effort is always oversupplied. Since enforcement is costly, the ("first-best") efficient approach to management by TAC would be to set a very small TAC and then to relax enforcement in order to allow a relatively large margin of non-compliance in order to achieve an efficient level of harvest. Because, as we have assumed, countries want to limit the extent of non-compliance, fully efficient TAC setting in this sense is not possible. 
We can see from (33) that if the two countries are identical (so that the TAC is efficiently allocated), we will have

$$
\lambda^{*}=\pi_{i h}-\omega_{i} \frac{\phi_{i Q}^{*}}{h_{i Q}^{*}}, \quad i=1,2,
$$

i.e., in each country the shadow price of the stock is equated with the marginal profit of harvest less the marginal cost of controlling harvest indirectly by means of adjustments in the TAC. ${ }^{11}$ If the quota shares $\gamma_{i}$ are such that the TAC is not efficiently allocated (which, in practice, is very likely to be the case), we will have

$$
\lambda^{*}=\frac{\sum_{i=1}^{2}\left[\pi_{i h} h_{i Q}^{*}-\omega_{i} \phi_{i Q}^{*}\right]}{\sum_{i=1}^{2} h_{i Q}^{*}}
$$

Here, condition (33) will hold with

$$
\pi_{i h}-\omega_{i} \frac{\phi_{i Q}^{*}}{h_{i Q}^{*}}>\lambda^{*}>\pi_{j h}-\omega_{j} \frac{\phi_{j Q}^{*}}{h_{j Q}^{*}}, \quad i \neq j .
$$

In this case, we can see that marginal social benefits are too large in Country $i$ and too small in Country $j$, given the various national parameters. All else equal, greater marginal social benefits are here implied, for example, by larger values of $\omega_{i}$ (which increases savings in enforcement costs) and smaller values of $c_{i}$ (which increases the marginal value of harvests). If it were politically feasible, of course, we could simply adjust the shares $\gamma_{i}$ and $\gamma_{j}$ so that the inequalities disappear and the TAC is efficiently allocated. Alternatively, we could conclude that (all else equal) Country $i$ cares too much about over-quota landings ( $\delta_{i}$ too large) and Country $j$ too little ( $\delta_{i}$ too small $)$.

The corresponding stock condition is

$$
\dot{\lambda}-r \lambda^{*}=-\mathcal{H}_{x}^{c} \equiv-\sum_{i=1}^{2}\left[\pi_{i x}-\omega_{i} \phi_{i x}^{*}-\lambda^{*} h_{i x}^{*}\right]-\lambda g_{x}
$$

\footnotetext{
$11 \overline{\text { Note that this is negative, since } \phi_{i Q}^{*}}<0$.
} 
where

$$
\pi_{i x} \equiv\left[p-\frac{c_{i}}{x} h_{i}^{*}\right] h_{i x}^{*}+\frac{c_{i}}{2 x^{2}} h_{i}^{* 2}>0
$$

as before, and

$$
\phi_{i x}^{*} \equiv \frac{\partial \phi_{i}^{*}(\cdot)}{\partial x}=\gamma_{i}^{2} Q^{2} \frac{\omega_{i} c_{i}}{x^{2} f_{i}^{2}}>0 .
$$

Assuming a steady state, we can find

$$
\lambda^{*}=\frac{\sum_{i=1}^{2}\left[\pi_{i x}-\omega_{i} \phi_{i x}^{*}\right]}{\sum_{i=1}^{2} h_{i x}^{*}-g_{x}+r}
$$

and hence, using (39),

$$
r=\left[g_{x}-\sum_{i=1}^{2} h_{i x}^{*}\right]+\frac{\sum_{i=1}^{2}\left[\pi_{i x}-\omega_{i} \phi_{i x}^{*}\right]}{\sum_{i=1}^{2}\left[\pi_{i h} h_{i Q}^{*}-\omega_{i} \phi_{i Q}^{*}\right] / \sum_{i=1}^{2} h_{i Q}^{*}} .
$$

With efficient quota allocation, we have

$$
r=\left[g_{x}-\sum_{i=1}^{2} h_{i x}^{*}\right]+\frac{\sum_{i=1}^{2}\left[\pi_{i x}-\omega_{i} \phi_{i x}^{*}\right]}{\pi_{i h}-\omega_{i} \phi_{i Q}^{*} / h_{i Q}^{*}}, \quad i=1,2,
$$

which can be compared with equation (17). Equation (46) differs from equation (17) in the appearance of $-\omega_{i} \phi_{i x}^{*}$ in the numerator, and the replacement of $\omega_{i} / h_{i \phi}^{*}$ by $\omega_{i} \phi_{i Q}^{*} / h_{i Q}^{*}$ in the denominator, of the "marginal stock effect", since now harvest is a function of national enforcement effort, which is a function of the TAC set by the central planner.

\subsection{Incentives to bid up a proposed TAC}

Consider an arbitrary TAC $\bar{Q}$, proposed either as the result of a stock assessment and scientific advice or based upon historical levels of exploitation. ${ }^{12}$ For the purposes of our discussion, let $\gamma_{i} \bar{Q}<\bar{h}_{i}, i=1,2$. We assume that the incentive for each country to try

12 In the Northeast Atlantic, scientific advice on fisheries is provided by ICES (the International Council for the Exploration of the Sea). In the case of EU fisheries, ICES advice is used by the European Commission to propose TACs, which must then be agreed by the Fisheries Council, comprising the fisheries ministers of all EU member states. Proposed TACs are either "advisory" - based on stock assessments - or "precautionary" - based only upon historic landings. 
to negotiate a higher TAC depends upon the (expected) increase in industry profits (net of any fine payments), less the associated enforcement costs, together with a subjective political cost incurred for increasing the TAC by appearing to be "anti-conservationist". Like $\delta_{i}(\cdot)$, the subjective cost of exceeding the quota, this could include damage to international reputation, as well as an internal (domestic) political cost. For simplicity, we again assume that this can be represented by a monetary cost, which we will in this case denote $\rho_{i}(Q ; \bar{Q}) \cdot{ }^{13}$

To be specific, let $\rho_{i}($.$) be a non-linearly increasing function of [Q-\bar{Q}]$, with

$$
\rho_{i}(\cdot) \equiv \frac{1}{2} \rho_{i}\left[\frac{Q^{2}-\bar{Q}}{\bar{Q}}\right] \Rightarrow \frac{\partial \rho_{i}(\cdot)}{\partial Q}=\rho_{i} \frac{Q}{\bar{Q}} .
$$

Given (33), we can find the marginal social benefit to Country $i$ as a function of the TAC $Q$ (for a given stock size $x$ ) as

$$
\frac{\partial S B_{i}}{\partial Q} \equiv\left[p-\frac{c_{i}}{x} h_{i}^{*}\right] h_{i Q}^{*}-\omega_{i} \phi_{i Q}^{*}
$$

Note that, while the disutility for over-quota harvest is implicit in the determination of $\phi_{i}^{*}(Q)$ and hence $h_{i}^{*}\left(\phi_{i}^{*}(Q)\right)$, it does not appear explicitly in the country's marginal benefit function since it does not represent a real resource cost. After substituting with (28), (30) and (35), this expression can be rearranged to yield

$$
\frac{\partial S B_{i}}{\partial Q}=\frac{x \delta_{i}^{2}}{c_{i} \gamma_{i}^{2} Q^{3}}+\gamma_{i}^{2} Q \frac{\omega_{i}^{2} c_{i}}{x f_{i}^{2}}>0,
$$

which is decreasing in $Q$ as we approach industry capacity harvest $\bar{h}_{i}$.

Straightforwardly, the incentive for Country $i$ to press for an increase in the TAC is

13 The alternative, but inevitably more complex, approach would be to specify a utility function containing arguments for both profits and political "cost". 
exhausted where

$$
\frac{x \delta_{i}^{2}}{c_{i} \gamma_{i}^{2} Q^{3}}+\gamma_{i}^{2} Q \frac{\omega_{i}^{2} c_{i}}{x f_{i}^{2}}=\rho_{i} \frac{Q}{\bar{Q}}
$$

Solving (50) for $Q$, we can find the (myopically) optimal negotiated TAC $\hat{Q}_{i}>\bar{Q}$ for Country $i$ as

$$
\hat{Q}_{i}=\sqrt[4]{\frac{x \delta_{i}^{2} / c_{i} \gamma_{i}^{2}}{\rho_{i} / \bar{Q}-\gamma_{i}^{2} \omega_{i}^{2} c_{i} / x f_{i}^{2}}}
$$

The desired TAC $\hat{Q}_{i}$ can be seen to be decreasing in the "political cost" parameter $\rho_{i}$, as we would expect, but increasing in the "compliance" parameter $\delta_{i}$ : all else equal, countries which care more about quota compliance have a greater incentive to seek a higher TAC, since their marginal gains reflect greater reductions in enforcement costs. It is also possible to show that $\hat{Q}_{i}$ is increasing in the TAC share $\gamma_{i}$. The finding that countries with larger TAC shares will seek a higher TAC may seem counter-intuitive. This is due, however, to the greater nominal gains for such countries together with the implicit assumption that the political cost (at least internationally) of seeking a higher TAC does not depend upon whether or not the country has a high or low TAC share. ${ }^{14}$

Voluntary agreement on the TAC would, we presume, be obtained if $\hat{Q}_{i}=\hat{Q}_{j}$ for Countries $i$ and $j$. Otherwise, either there must be an external arbiter to impose a decision, or, given that the $\gamma_{i}$ are assumed fixed, agreement requires countries to change their "preferences for sustainability", which implies changing their values of $\rho_{i}(!)$. Alternatively, there could be side payments, or de facto marginal adjustments of TAC shares by means of quota exchanges. $^{15}$

\footnotetext{
14 We could, alternatively, suppose that those countries with higher TAC shares would have a greater political stake in the sustainability of the resource and hence a larger value of $\rho_{i}$.

15 Such ad hoc exchanges of quota are increasingly conducted between EU Member States, for example, following
} 


\section{Numerical simulation}

Here we present some results from a set of numerical simulations. In order to keep things relatively simple and tractable, we assume symmetrical countries and equal quota shares. Our principle focus here is on the implications for efficient harvesting of indirect control of harvest through quota regulation.

\subsection{Stock growth function and economic model parameters}

We specify the stock growth function as

$$
g(x) \equiv \alpha x \ln \frac{k}{x}
$$

where $\alpha$ and $k$ are parameters. The marginal growth rate is then

$$
g_{x}=\alpha\left[\ln \frac{k}{x}-1\right]
$$

With $\alpha=0.30$ and $k=100,000$ tonnes, the annual maximum sustainable yield (MSY) is given by $\alpha k / e=\mathbf{1 1 , 0 4 0}$ tonnes, where the stock size $x$ is equal to $k / e=\mathbf{3 6 , 7 9 0}$ tonnes. ${ }^{16}$

The parameter values for the economic models are as follows.

the December Fisheries Council meeting at which the TACs for the following year are decided.

16 All results are rounded to the nearest 10 tonnes or 100,000 euros. 


\begin{tabular}{|c|c|c|}
\hline Market price $(€ 000)$ & $p_{i}$ & 2.0 \\
\hline Harvest cost parameter $(€ 000)$ & $c_{i}$ & 5.0 \\
\hline Fine rate $(€ 000)$ & $f_{i}$ & 7.5 \\
\hline Enforcement cost parameter $(€ 000)$ & $\omega_{i}$ & 5.0 \\
\hline TAC shares & $\gamma_{i}$ & 0.5 \\
\hline Interest rate & $r$ & $5 \%$ \\
\hline
\end{tabular}

If harvest is unregulated, there is no steady state solution. At a stock size of 30,000 tonnes, for example, capacity harvest by both countries totals 24,000 tonnes, more than double the sustainable yield of 10,840 tonnes. Without regulation, the fishery would collapse.

\subsection{Efficient harvest}

We can find the efficient harvest (i.e., the level of harvest a social planner would choose if she could mandate harvest without the need for enforcement) as the solution to the steady state equations

$$
g_{x}+\frac{\sum_{i=1}^{2} \pi_{i x}}{\pi_{i h}}-r=0, \quad i=1,2
$$

and

$$
\sum_{i=1}^{2} h_{i}^{*}=g(x)
$$

where

$$
\pi_{i x}=\frac{\partial \pi_{i}}{\partial x}=\frac{c_{i}}{2 x^{2}} h_{i}^{* 2}, \quad \pi_{i h}=\frac{\partial \pi_{i}}{\partial h_{i}}=p-\frac{c_{i}}{x} h_{i}^{*}
$$

Given the parameters of our simulation model, we obtain the efficient (steady-state) total 
annual harvest as 11,000 tonnes, shared equally between the two countries. The corresponding stock size is $\mathbf{3 9 , 7 5 0}$ tonnes. The total annual rents generated in the fishery are given by

$$
\sum_{i=1}^{2} \pi_{i}\left(h_{i}, x\right)=\sum_{i=1}^{2} h_{i}^{*}\left[p-\frac{c_{i}}{2 x} h_{i}^{*}\right],
$$

which in this case amount to $€ \mathbf{1 8 . 2 m}$.

\subsection{Efficient enforcement}

The efficient levels of enforcement $\phi_{i}^{*}$ in each country satisfy the steady state equations

$$
\left[g_{x}-\sum_{i=1}^{2} h_{i x}^{*}\right]+\frac{\sum_{i=1}^{2} \pi_{i x}}{\pi_{i h}-\omega_{i} / h_{i \phi}^{*}}-r=0, \quad i=1,2,
$$

(which are implicit functions of $\phi_{i}$ and $x$ ) together with the stock constraint. Here,

$$
h_{i x}^{*}=\frac{\partial h_{i}^{*}}{\partial x}=\frac{1}{c_{i}}\left[p-\phi_{i}^{*} \frac{f_{i}}{q_{i}}\right], \quad h_{i \phi}^{*}=\frac{\partial h_{i}^{*}}{\partial \phi_{i}}=-\frac{x f_{i}}{c_{i} q_{i}},
$$

with

$$
\pi_{i x}=\frac{\partial \pi_{i}}{\partial x}=\left[p-\frac{c_{i}}{x} h_{i}^{*}\right] h_{i x}^{*}+\frac{c_{i}}{2 x^{2}} h_{i}^{* 2}, \quad \pi_{i h}=\frac{\partial \pi_{i}}{\partial h_{i}}=p-\frac{c_{i}}{x} h_{i}^{*} .
$$

Suppose regulators set a (conservative) TAC of 9,000 tonnes, which translates into two national quotas $q_{i}$ of 4,500 tonnes each. Then the efficient levels of enforcement are found to be equivalent to 0.066 or a $6.6 \%$ risk of detection in each country. The total annual harvest is $\mathbf{1 0 , 8 4 0}$ tonnes (around $20 \%$ over quota) with the stock size at $\mathbf{3 0 , 0 3 0}$ tonnes.

Given the TAC, with efficient enforcement annual total rents are $€ \mathbf{1 6 . 8 m}$, but the total cost of enforcement is $€ \mathbf{6 . 6 m}$, reducing the total net economic value of the fishery to $€ 10.2 \mathrm{~m}$. 
We can do better than this, however, by setting a lower TAC. With a TAC of $\mathbf{7 , 0 0 0}$ tonnes, for example, the total harvest is $\mathbf{1 0 , 9 6 0}$ tonnes from a stock of $\mathbf{3 2 , 6 1 0}$ tonnes. Total rents are now $€ \mathbf{1 7 . 3 m}$ and enforcement costs are reduced to $€ \mathbf{5 . 4 m}$, giving a net value of $€ \mathbf{1 1 . 9 m}$. A lower TAC results in a lower level of enforcement (now just $5.4 \%$ ) which allows fleets to increase their quota non-compliance margin to $57 \%$.

\subsection{Myopic enforcement}

Under myopic enforcement, as we have seen, only the assumption of a positive "disutility for over-quota harvest" parameter $\delta_{i}$ ensures that a country expends any enforcement effort. Given national quotas of 4,500 tonnes, a value for $\delta_{i}$ of 7.19 in each country will, in our model, achieve an efficient level of enforcement effort.

\subsection{An efficient TAC}

The efficient TAC $Q^{*}$ is found by solving the implicit equations

$$
\left[g_{x}-\sum_{i=1}^{2} h_{i x}^{*}\right]+\frac{\sum_{i=1}^{2}\left[\pi_{i x}-\omega_{i} \phi_{i x}^{*}\right]}{\pi_{i h}-\omega_{i} \phi_{i Q}^{*} / h_{i Q}^{*}}-r=0, \quad i=1,2,
$$

together with the stock constraint. In this case, we have

$$
h_{i}^{*}=\frac{x}{c_{i}}\left[p-\frac{\delta_{i}}{\gamma_{i} Q}\right]+\gamma_{i} Q \frac{\omega_{i}}{f_{i}},
$$

while

$$
h_{i x}^{*}=\frac{\partial h_{i}^{*}}{\partial x}=\frac{1}{c_{i}}\left[p-\frac{\delta_{i}}{\gamma_{i} Q}\right], \quad h_{i Q}^{*}=\frac{\partial h_{i}^{*}}{\partial Q}=\frac{\delta_{i} x}{\gamma_{i} Q^{2} c_{i}}+\gamma_{i} \frac{\omega_{i}}{f_{i}},
$$

with

$$
\phi_{i x}^{*}=\frac{\partial \phi^{*}}{\partial x}=\gamma_{i}^{2} Q^{2} \frac{\omega_{i} c_{i}}{x^{2} f_{i}^{2}}, \quad \phi_{i Q}^{*}=\frac{\partial \phi^{*}}{\partial Q}=-2 \gamma_{i}^{2} Q \frac{\omega_{i} c_{i}}{x f_{i}^{2}}
$$


To begin with, let us assume that each country has a $\delta_{i}$ value equal to that which produced an efficient level of enforcement given a TAC of 9,000 tonnes $\left(\delta_{i}=7.19\right)$. The optimal TAC is then found to be $\mathbf{8 , 1 5 0}$ tonnes. With this TAC, each country's fleet harvests 5,140 tonnes, exceeding their quota by a $26 \%$ margin, in response to an enforcement effort (risk of detection) of $8.2 \%$. The steady state harvest is then $\mathbf{1 0 , 2 7 0}$ tonnes and the stock size is $\mathbf{5 1 , 3 0 0}$ tonnes. Now total rents are $€ \mathbf{1 8 . 0 m}$, but enforcement costs are $€ \mathbf{8 . 1} \mathbf{m}$, giving a net fishery value of $€ \mathbf{9 . 8 m}$.

We could suppose, alternatively, that countries wanted more strictly to limit the extent of over-quota harvest, say to a margin of $5 \%$. In our model, this would require $\delta_{i}=8.95$. The optimal TAC is now $\mathbf{9 , 8 8 0}$ tonnes with a total harvest of $\mathbf{1 0 , 3 7 0}$ tonnes from a steady state stock of $\mathbf{5 0 , 2 7 0}$ tonnes. This requires enforcement effort equivalent to $9.8 \%$, which costs $€ \mathbf{9 . 8 m}$ compared to industry rents of $€ \mathbf{1 8 . 1} \mathbf{m}$, reducing the net value of the fishery to $€ 8.3 \mathrm{~m}$.

What happens if we relax the countries' attitudes towards compliance? Suppose that the value of $\delta_{i}$ is reduced to $\delta_{i}=3.00$ in each country. Now, we find that the optimal TAC is only 3,660 tonnes, although the total harvest is little changed at $\mathbf{1 0 , 0 8 0}$ tonnes $(175 \%$

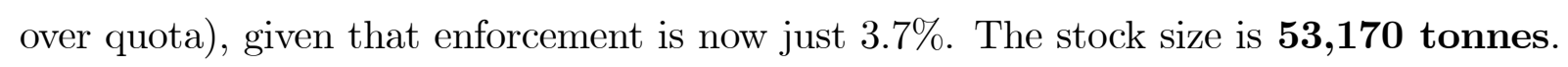
Total rents are $€ \mathbf{1 7 . 8 m}$, but enforcement costs are only $€ \mathbf{3 . 7} \mathbf{m}$, giving a total net value of $€ 14.0 \mathrm{~m}$.

Now suppose that countries care very little about their fleets' compliance. Let $\delta_{i}=0.10$. In this case the optimal TAC is just 130 tonnes. The level of enforcement is around 
$0.1 \%$ in each country, permitting a total harvest of $\mathbf{1 0 , 0 2 0}$ tonnes (a relative quota violation of $7,600 \%$ !). The steady state stock size is $\mathbf{5 3 , 7 1 0}$ tonnes. Total industry rents are $€ \mathbf{1 7 . 7 m}$, but with enforcement costs of little over $€ \mathbf{0 . 1 m}$ the total net value of the fishery is $€ \mathbf{1 7 . 6 m}$.

\section{Conclusion}

We have analysed the exploitation of a shared fish stock by two countries with fixed shares of a TAC and imperfect control over their fishing fleets' quota compliance. We have in mind an international system of fisheries governance such as is encountered, on a larger scale, in the EU. Given the harvest response function of its fishing industry, each country is assumed to choose an amount of costly enforcement effort in order to maximise current period economic benefits, subject to what it considers an acceptable degree of compliance with the national quota. The resulting level of enforcement is only efficient for the quotas in force if each country's relative "disutility" for over-quota landings equates with the shadow price of the stock under optimal enforcement. Otherwise, we have the possibility that a country will expend too much costly enforcement effort, as well as the possibility of too little. Excessive costly enforcement implies a harvest level that is too low, even though it may be closer to the quota set for the country concerned. Similarly, "first-best" quotas (determined, perhaps, under the assumption of perfect and costless compliance) will be too high when we take into account both non-compliance and the costs of enforcement, as the numerical results clearly demonstrate.

Solving simultaneously the best-response harvest and enforcement choices of the countries 
and their fishing fleets, we found harvest as a direct function of the TAC. We then considered optimal management of the fishery using the TAC as the control variable. Because enforcement is costly, the most efficient solution would be to set the TAC at a very low level and to then use a minimal amount of enforcement in order to reach an efficient level of harvest by permitting a relatively large degree of quota non-compliance. In our model, this is limited by the countries' disutility for over-quota harvests (which nevertheless ensures that there is some enforcement effort). While setting a small TAC and relaxing enforcement may be rational, as the numerical simulations show, it is not (to our knowledge) an approach that is observed in practice. The reasons for this are almost certainly political, but a possible economic justification is that there are significant non-pecuniary (normative and social) incentives for quota compliance and that reducing the quota and enforcement to induce a greater margin of illegal landings may result in a serious loss of legitimacy for quota regulations per $s e{ }^{17}$ This analysis of the TAC setting process is a major contribution of the paper, as it clearly diverges from the usual modelling of the TAC as optimal harvest, whether in economic or purely biological terms. ${ }^{18}$

Finally, we considered briefly the incentives for countries to seek increases in the TAC above the level recommended during the annual TAC negotiations. In our analysis, the only brake on seeking a higher TAC is provided by an assumed political cost for appearing "anti-conservationist". We could also interpret this as a real concern for resource conservation, but experience of TAC negotiations in Europe would suggest that our char-

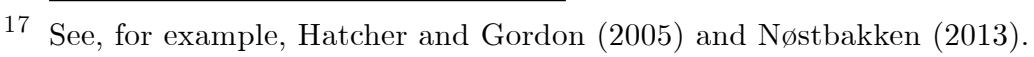

18 This is not the same, of course, as TACs which are deliberately set at a lower level in order to allow for discards or undeclared landings. In order to keep our model relatively simple we have ignored the distinction between catches and landings. 
acterisation is not unrealistic. We find, interestingly, that countries which care more about quota compliance gain proportionately more from increases in their quotas, since they benefit from greater reductions in enforcement costs, and hence, all else equal, may argue more strongly for increases in the TAC. 


\section{References}

Beard, R., and L. Nøstbakken (2010). Multi-jurisdiction quota enforcement for transboundary renewable resources. SNF Working Paper No. 28/10. Bergen: SNF.

Clark, C. W., and G. Munro (1975). The economics of fishing and modern capital theory. Journal of Environmental Economics and Management, 2: 92-106.

Conrad, J. M., (1999). Resource Economics. Cambridge: Cambridge University Press.

Hannesson, R., (2011). Game theory and fisheries. Annual Review of Resource Economics, 3: $181-202$.

Hatcher, A., (2005). Non-compliance and the quota price in an ITQ fishery. Journal of Environmental Economics and Management, 49: 427-436.

Hatcher, A., (2012). Market power and compliance with output quotas. Resource and Energy Economics, 34: 255-269.

Hatcher, A., and D. Gordon (2005). Further investigations into the factors affecting compliance with UK fishing quotas. Land Economics, 81: 71-86.

Holden, M., and D. Garrod (1994). The Common Fisheries Policy. Oxford: Blackwell (Fishing News Books). 
Kaitala, V., and M. Lindroos (2007). Game theoretic applications to fisheries. In: A. Weintraub, ed., Handbook of Operations Research in Natural Resources. Berlin: Springer.

Lindroos, M., (2008). Coalitions in international fisheries management. Natural Resource Modeling, 21: 366-384.

Lindroos, M., and G. Munro (2013). Introduction to the special issue on game theory and fisheries: recent issues. Strategic Behavior and the Environment, 3: 1-5.

Long, N. V., (2011). Dynamic games in the economics of natural resources: a survey. Dynamic Games and Applications, 1: 115-148.

Long, N. V., (2012). Applications of dynamic games to global and transboundary environmental issues: a review of the literature. Strategic Behavior and the Environment, 2: $1-59$.

Nøstbakken, L., (2013). Formal and informal quota enforcement. Resource and Energy Economics, 35: 191-215.

Munro, G., (2009). Game theory and the development of resource management policy: the case of international fisheries. Environment and Development Economics, 14: 7-27.

Sutinen, J. G., and P. Andersen (1985). The economics of fisheries law enforcement. Land Economics, 61: 387-397. 